\title{
Obesity and insulin resistance
}

\author{
Barbara B. Kahn and Jeffrey S. Flier \\ Division of Endocrinology and Metabolism, Department of Medicine, Beth Israel Deaconess Medical Center and Harvard Medical School, \\ Boston, Massachusetts, USA \\ Address correspondence to: Barbara B. Kahn, Division of Endocrinology and Metabolism, Beth Israel Deaconess Medical Center, \\ 99 Brookline Avenue, Boston, Massachusetts 02215, USA. Phone: (617) 667-5422; Fax: (617) 667-2927; E-mail: bkahn@caregroup.harvard.edu.
}

The association of obesity with type 2 diabetes has been recognized for decades, and the major basis for this link is the ability of obesity to engender insulin resistance. Insulin resistance is a fundamental aspect of the etiology of type 2 diabetes and is also linked to a wide array of other pathophysiologic sequelae including hypertension, hyperlipidemia, atherosclerosis (i.e., the metabolic syndrome, or syndrome $\mathrm{X}$ ), and polycystic ovarian disease (1). Although many details of the mechanisms by which the enlarged adipose tissue mass that defines obesity causes systemic insulin resistance remain unknown, the past several years have witnessed an explosive increase in our understanding of what may now be referred to as the adipo-insulin axis. There are also grounds for considering the related possibility that insulin resistance and hyperinsulinemia, in addition to being caused by obesity, can contribute to the development of obesity. In this Perspective, we will review recent progress, highlight areas of controversy or uncertainty, and suggest approaches to clarifying the unresolved issues.

Insulin action in the adipocyte. Insulin is a critical regulator of virtually all aspects of adipocyte biology, and adipocytes are one of the most highly insulin-responsive cell types. Insulin promotes adipocyte triglyceride stores by a number of mechanisms, including fostering the differentiation of preadipocytes to adipocytes and, in mature adipocytes, stimulating glucose transport and triglyceride synthesis (lipogenesis), as well as inhibiting lipolysis (Figure 1). Insulin also increases the uptake of fatty acids derived from circulating lipoproteins by stimulating lipoprotein lipase activity in adipose tissue. Insulin's metabolic effects are mediated by a broad array of tissue-specific actions that involve rapid changes in protein phosphorylation and function, as well as changes in gene expression. The fundamental biologic importance of these actions of insulin is evidenced by the fact that the insulin signaling cascade which initiates these events is largely conserved in evolution from C. elegans to humans (2).

The initial molecular signal for insulin action involves activation of the insulin receptor tyrosine kinase, which results in phosphorylation of insulin receptor substrates (IRSs) on multiple tyrosine residues. These phosphotyrosine residues act as docking sites for many SH2 domain-containing proteins, including the $\mathrm{p} 85$ regulatory subunit of phosphoinositide $3^{\prime}$ kinase (PI3K). Binding of the p110 catalytic subunit of PI3K to p85 activates the lipid kinase that promotes glucose transport (3). Whereas activation of PI3K is necessary for full stimulation of glucose transport by insulin, emerging evidence suggests that it is not sufficient and another pathway may also be necessary $(4,5)$. The signals downstream of PI3K are still unknown, and there is controversy as to whether the serine/threonine kinase Akt/protein kinase $\mathrm{B}(\mathrm{PKB})(6,7)$ or the protein kinase $\mathrm{C}(\mathrm{PKC})$ isoform $\lambda / \zeta(8)$ mediates insulin stimulation of glucose transport. Most likely, the pathways that mediate insulin's metabolic effects diverge downstream of PI3K $(7,9)$ and show differential sensitivity to varying levels of insulin. For example, the antilipolytic effect of insulin requires much lower insulin concentrations than stimulation of glucose transport. Hence, even in insulinresistant states in which glucose transport is impaired, sensitivity to insulin's antilipolytic effect is relatively preserved, resulting in maintenance or expansion of adipose stores. Insulin also activates the ras-mitogen-activated protein kinase (ras-MAPK) signaling cascade. This pathway appears to be important for the mitogenic effects of insulin, but most data do not implicate the MAPK pathway in the well-studied metabolic actions of insulin. Insulin action in adipocytes also involves changes in gene transcription. The transcription factor ADD-1/SREBP-1c (adipocyte determination and differentiation factor-1/sterol regulatory element-binding protein-1c) may play a critical role in the actions of insulin to regulate adipocyte gene expression (10-12), by inducing genes involved in lipogenesis and repressing those involved in fatty acid oxidation. Transcription factors of the forkhead family may also play a major role in transducing insulin signals to the nucleus (13). The relative functions of the ADD-1/SREBP-1c and forkhead pathways should be the subject of future research.

Insulin resistance in obesity and type 2 diabetes. The term "insulin resistance" usually connotes resistance to the effects of insulin on glucose uptake, metabolism, or storage. Insulin resistance in obesity and type 2 diabetes is manifested by decreased insulin-stimulated glucose transport and metabolism in adipocytes and skeletal muscle and by impaired suppression of hepatic glucose 
output (1). These functional defects may result, in part, from impaired insulin signaling in all three target tissues and, in adipocytes, also from downregulation of the major insulin-responsive glucose transporter, GLUT4. In both muscle and adipocytes, insulin binding to its receptor, receptor phosphorylation and tyrosine kinase activity, and phosphorylation of IRSs are reduced. There are also tissue-specific alterations: In adipocytes from obese humans with type 2 diabetes, IRS-1 expression is reduced, resulting in decreased IRS1 -associated PI3K activity, and IRS- 2 becomes the main docking protein for PI3K (14). In contrast, in skeletal muscle of obese, type 2 diabetic subjects, IRS- 1 and IRS2 protein levels are normal but PI3K activity associated with both IRSs is impaired (15).

One mechanism for the signaling defects in obesity may be the increased expression and activity of several protein tyrosine phosphatases (PTPs), which dephosphorylate and thus terminate signaling propagated through tyrosyl phosphorylation events. Some data indicate that at least three PTPs, including PTP1B, leukocyte antigen-related phosphatase (LAR), and src-homologyphosphatase 2, are increased in expression and/or activity in muscle and adipose tissue of obese humans and rodents (16). PTP1B and LAR have been shown to dephosphorylate the insulin receptor and IRS-1 in vitro $(16,17)$. In fact, mice in which PTP1B has been knocked out have increased insulin sensitivity and resistance to diet-induced obesity $(18,19)$, at least in part, due to increased energy expenditure (19). This suggests a regulatory role for PTP1B not only in insulin action, but also in energy homeostasis. Interestingly, the insulin sensitivity is present in muscle and liver but not in adipocytes $(18,19)$. Whether there is a causal relationship between the insulin sensitivity and leanness/energy expenditure or whether these are regulated by independent signaling pathways is a key question.

Other mechanisms also contribute to insulin resistance in obesity. In morbid obesity, the expression of various insulin signaling molecules is reduced in skeletal muscle (20). In all forms of obesity and diabetes, a major factor contributing to the impaired insulin-stimulated glucose transport in adipocytes is the downregulation of GLUT4. However, in skeletal muscle of obese and diabetic humans, GLUT4 expression is normal (reviewed in ref. 21) and defective glucose transport appears to be due to impaired translocation, docking, or fusion of GLUT4-containing vesicles with the plasma membrane.

Although insulin resistance is characteristic of obesity and type 2 diabetes, it is not established that all of insulin's actions are impaired in individuals with both conditions. It is possible that hepatic lipogenesis and lipid storage are being driven to excess in adipose tissue, whereas other insulin effects related to glucose homeostasis are impaired. It will be important to identify the signaling pathways and transcription factors that could allow for such discordant actions of insulin.

\section{Reduced glucose disposal into adipose tissue in obesity}

The action of insulin to lower blood glucose levels results from suppression of hepatic glucose production and increased glucose uptake into muscle and fat. Muscle has long been considered the major site of insulin-stimulated glucose uptake in vivo, with adipose tissue contributing relatively little to total body glucose disposal. Support for this conclusion comes from the fact that measurements of 2-deoxyglucose uptake in vivo show at least ten times more glucose per milligram of tissue going into muscle than into white adipose tissue (WAT) (22). Because muscle mass is considerably greater than WAT mass, at least in lean rodents and humans, this observation has been taken to indicate the prominent contribution of muscle to glucose disposal. Although glucose transport into brown adipose tissue (BAT) is higher than in many muscle groups, the mass of BAT is small even in rodents, making this an unlikely site to account for large amounts of total body glucose uptake. Thus, it has been viewed as unlikely that diminished glucose uptake into fat could account for diminished whole body glucose uptake in obesity. However, transgenic studies have raised the possibility of a greater role for glucose uptake into fat in systemic glucose homeostasis than was previously believed. Overexpression of GLUT4 selectively in fat enhances whole body insulin sensitivity and glucose tolerance (23) even in overtly diabetic mice (24). Furthermore, knocking out GLUT4 selectively from fat results in a degree of insulin resistance similar to that seen with muscle-specific knockout of GLUT4 (unpublished observation). Whether this systemic insulin resistance results directly from the absence of adipose glucose transport or indirectly from possible effects of altered glucose uptake on the release of other molecules from adipocytes remains a key question. Likely candidates for indirect effects are FFA, leptin, or TNF- $\alpha$, all of which are known to affect glucose homeostasis (see below). Undoubtedly there are other, as yet undiscovered, molecules from fat that influence systemic metabolism.

Further support for a potential direct role of adipocytes in regulating systemic glucose homeostasis comes from studies in which rodents or humans are treated with the $\beta 3$ adrenergic agonist CL316,243 $(25,26)$. Since $\beta 3$ adrenergic receptors are expressed almost exclusively in fat, effects of these agents would be expected to be initiated by alterations in fat. Treatment with CL316,243 results in enhanced sensitivity of both whole body glucose uptake and suppression of hepatic glucose production (25). These effects are accompanied by increased glucose uptake in adipose tissue with no effect in multiple muscle groups studied. Thus, increasing glucose uptake selectively in fat with $\beta 3$ adrenergic receptor agonists may improve whole body glucose uptake, with the effects in fat 


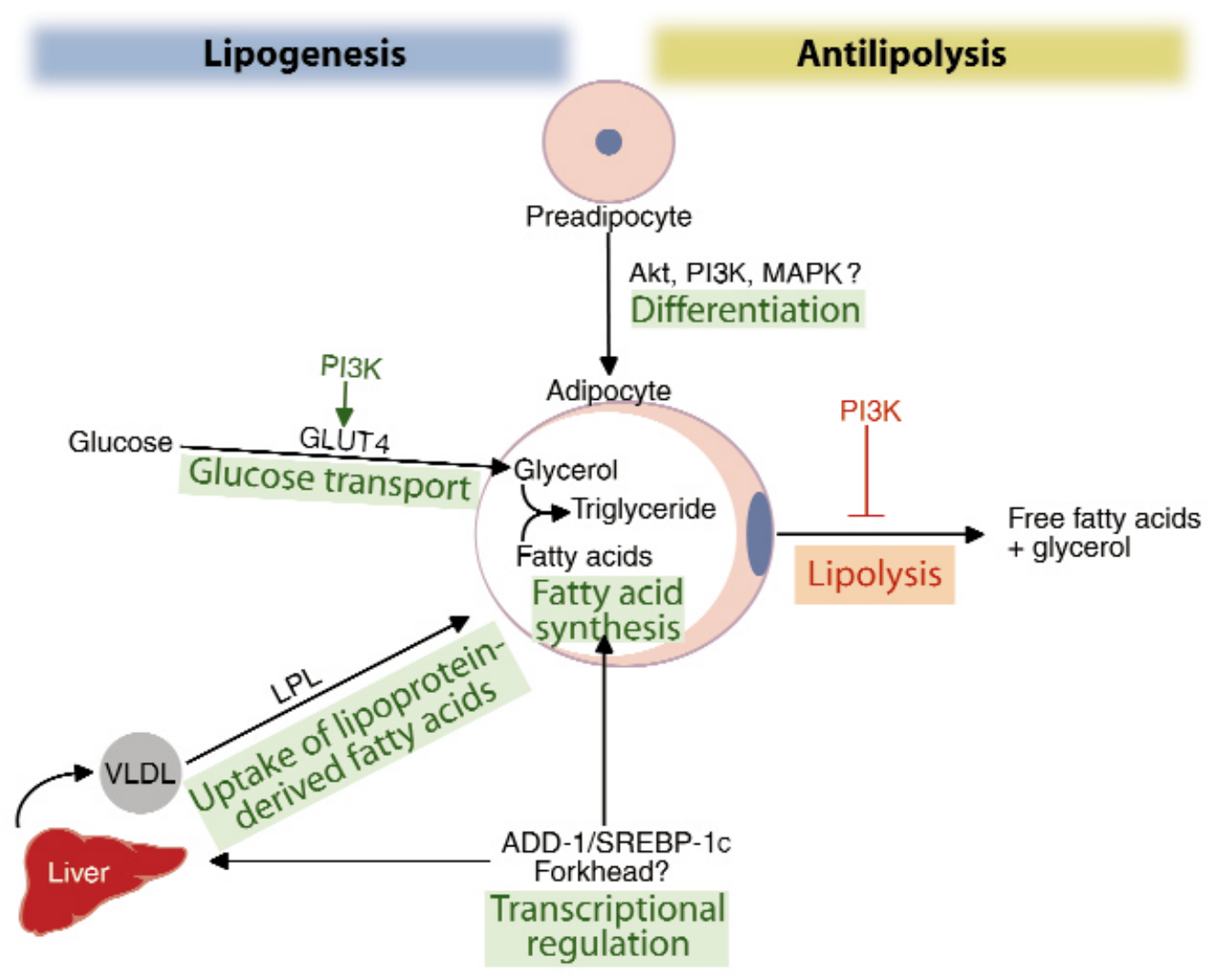

Figure 1

Pleiotropic effects of insulin to promote adipose storage. Insulin stimulates differentiation of preadipocytes to adipocytes. In adipocytes, insulin promotes lipogenesis by stimulating the uptake of glucose and lipoprotein-derived fatty acids and by inducing ADD-1/SREBP-1c, which regulates genes promoting fatty acid synthesis and lipogenesis, not only in adipocytes but also in hepatocytes. Insulin may also regulate transcription through Forkhead transcription factors. Insulin diminishes triglyceride breakdown by inhibiting lipolysis. Many of these metabolic pathways are regulated by the PI3K signaling pathway.

indirectly resulting in increased insulin sensitivity in liver. Alternatively, $\beta 3$ agonists may work by changing the release of some adipocyte product that influences systemic insulin sensitivity.

The significance of the location of body fat for insulin resistance. The relationship between obesity and insulin resistance is seen across all ethnic groups and is evident across the full range of body weights. Large epidemiologic studies reveal that the risk for diabetes, and presumably insulin resistance, rises as body fat content (measured by body mass index [BMI]) increases from the very lean to the very obese, implying that the "dose" of body fat has an effect on insulin sensitivity across a broad range (27). Although this relationship is seen with measures of adiposity such as BMI, which reflect general adiposity, it is critical to realize that all sites of adiposity are not equal in this regard. Central (intra-abdominal) depots of fat are much more strongly linked to insulin resistance, type 2 diabetes, and cardiovascular disease than are peripheral (gluteal/subcutaneous) fat depots (28). This fact about fat and insulin sensitivity has not been ade- quately explained. It is possible that an unknown common factor, either genetic or environmental, produces both insulin resistance and the central pattern of regional adiposity, and that central obesity does not actually cause insulin resistance. Alternatively, some biochemical feature of intra-abdominal adipocytes may directly influence systemic insulin sensitivity.

A leading hypothesis in this regard is that intraabdominal adipocytes are more lipolytically active, in part due to their complement of adrenergic receptors. This would increase intraportal FFA levels and flux, which might inhibit insulin clearance and promote insulin resistance by mechanisms that are still uncertain. Hyperinsulinemia per se can cause insulin resistance by downregulating insulin receptors and desensitizing postreceptor pathways, as was confirmed by overexpression of insulin in livers of otherwise normal transgenic mice. This transgene resulted in an age-related reduction in insulin receptor expression, glucose intolerance, and hyperlipidemia without any primary genetic defect in insulin action or secretion (reviewed in ref. 29). An alternative hypothesis is that, since 
adipocytes are now known to secrete many factors that are capable of exerting systemic effects (see below), the array of factors secreted by intra-abdominal adipocytes may be particularly harmful to systemic insulin sensitivity. So far, this hypothesis remains unproven.

Adipocytes as endocrine cells. Adipocytes are well known for their essential role as energy storage depots for triglycerides, from which energy is called forth at times of need in the form of FFAs and glycerol. However, data emerging over the past several years have established an additional role for the adipocyte, that of secretory cell (Figure 2). Adipocytes express and secrete numerous peptide hormones and cytokines, including TNF- $\alpha$; plasminogen-activator inhibitor-1, which helps maintain hemostasis; angiotensinogen, whose proteolytic product regulates vascular tone; and leptin, which plays a central role in regulating energy balance. Adipose tissue can also produce active steroid hormones, including estrogen and cortisol $(30,31)$. Through such secreted products, adipocytes possess the capacity to influence local adipocyte biology, as well as systemic metabolism at sites as diverse as brain, liver, muscle, $\beta$ cells, gonads, lymphoid organs, and systemic vasculature. This realization raises many possibilities for additional links between adipose function or mass and insulin resistance, independent of the adipocyte's roles in energy storage and release (Figure 1).

A great deal of interest has followed the discovery that adipocytes express and secrete the cytokine TNF$\alpha$, and that enlarged adipocytes from obese animals and humans overexpress this factor (32). Although not all studies have found TNF- $\alpha$ to be elevated in obesity, the normal circulating levels of this factor are at the limit of detection, making quantitative analysis uncertain. This low expression may indicate that
TNF- $\alpha$ acts in a paracrine rather than endocrine fashion. Alternative approaches to assessing a role of TNF- $\alpha$ in systemic insulin resistance are therefore needed, and in some but not all studies using neutralizing antibodies or other agents to block TNF- $\alpha$ function in animal models, it appears that such blockade heightens insulin sensitivity (33).

TNF- $\alpha$ has many effects on adipocyte function, and these include actions to inhibit lipogenesis and to increase lipolysis. These actions have been viewed by some as a feedback loop against excessive energy storage. Can excessive TNF- $\alpha$ cause insulin resistance? TNF- $\alpha$ signaling impairs insulin signaling, in part through serine phosphorylation of IRS-1 $(32,33)$, and can reduce GLUT4 gene expression, so a plausible cellular basis for TNF- $\alpha$ as a mediator of insulin resistance has been established. Further support derives from the beneficial effect of knockout of TNF $\alpha$ or TNF $\alpha$ receptor genes on insulin resistance in several animal models of obesity-associated insulin resistance $(32,33)$. However, improvement of insulin resistance in response to loss of TNF signaling is at best partial, and the effect of TNF neutralization has not been seen in all experimental models. Thus, TNF- $\alpha$ may be a partial contributor to insulin resistance, but other factors must exist.

Leptin, the product of the $o b$ gene, may be one such factor. This adipocyte-derived hormone exerts pleiotropic effects, including profound effects on satiety, energy expenditure, and neuroendocrine function (34). The most compelling role of leptin from an evolutionary standpoint is its capacity to serve as a bidirectional signal that switches metabolic physiology and neuroendocrine status between programs appropriate to the fed and starved states. The proposed role for rising leptin as a strong (adipostatic) signal to prevent obesity is easily

\section{Inert Storage Depot}

\section{Figure 2}

Evolving view of the biological functions of the adipocyte. Previously, adipocytes were considered to be inert storage depots releasing fuel as fatty acids and glycerol in time of fasting or starvation. More recently it has become clear that adipocytes are endocrine glands that secrete important hormones, cytokines, vasoactive substances, and other peptides. ANS, autonomic nervous system.

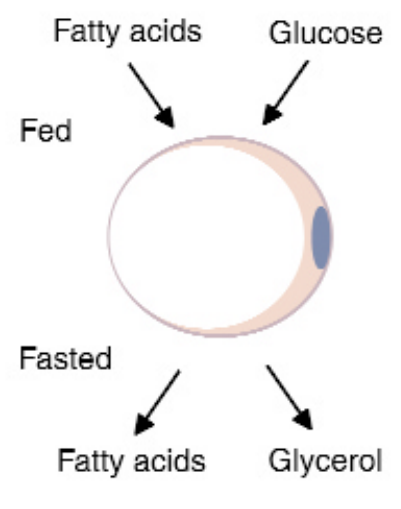

\section{Secretory/Endocrine Gland}

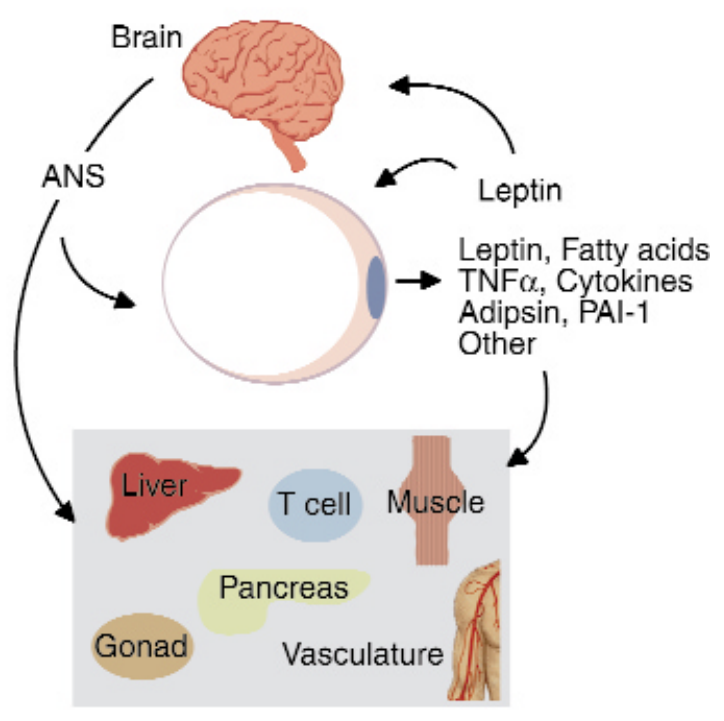




\section{Figure 3}

Leptin exerts multiple actions to regulate glucose homeostasis through autocrine, paracrine, endocrine, and neural circuits. Whereas many of leptin's effects are mediated by the CNS, some effects may be exerted directly at the level of insulin target tissues or pancreatic islet cells.

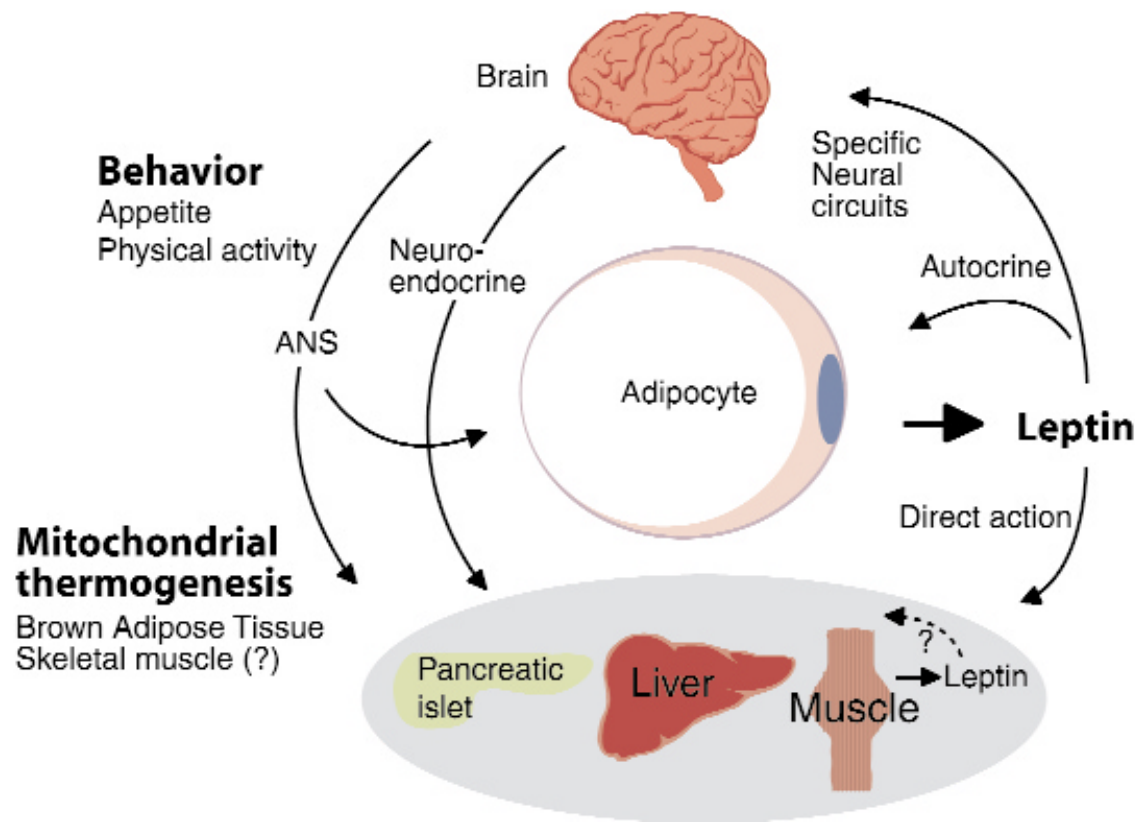

subverted by leptin resistance. Since increased energy stores would favor survival in periods of famine, the adipostatic aspect of leptin action may have been selected against during the course of evolution (35). This view of leptin as being primarily involved in the starvation/feeding switch does not negate the fact that the absence of leptin in both rodents and humans produces severe obesity for which leptin is clearly the cure. Nor does it lessen the importance of determining the molecular basis for leptin resistance, which limits the capacity of rising leptin to prevent obesity in most situations.

Severe insulin resistance is a well known feature of deficiency of leptin or its receptor in the $a b / o b$ or $d b / d b$ mouse strains, and these models were among the first to be investigated for the pathogenesis of insulin resistance in the early 1970s. Insulin resistance and hyperinsulinemia occur early in the life of these animals, are out of proportion to their adiposity at early stages, and exceed the insulin resistance and hyperinsulinemia due simply to hyperphagia and obesity. The degree to which diabetes (as opposed to insulin resistance without hyperglycemia) develops in these mice is determined by their genetic background, via effects on insulin secretory capacity and possibly other factors. The identity of these background modifier genes is unknown at present. Leptin's major site of action is the hypothalamus, especially in selected nuclei within the ventrobasal hypothalamus, where neurons that are directly regulated by leptin reside (36). The fact that hyperinsulinemia and insulin resistance are produced by hypothalamic lesions affecting the ventromedial hypothalamic nucleus suggested a major role for the central nervous system (CNS) in regulation of insulin action or secretion. Consistent with this model, cur- rent evidence suggests that leptin exerts much of its effect on metabolism and satiety through actions within the ventrobasal hypothalamus.

The result of leptin replacement in $o b / o b$ mice on diabetes and insulin resistance is dramatic. Leptin treatment causes both glucose and insulin levels to fall within hours of administration, before changes in either food intake or body weight occur, and prolonged leptin has effects on glucose and insulin that exceed those seen in pair-fed $o b / o b$ mice (37). Leptin has a clear insulin-sensitizing effect acutely and also after chronic administration to normal rodents (37-39). The molecular basis for the insulin-sensitizing effect of leptin remains a topic of great interest. Two general views prevail. According to one, the metabolic actions of leptin are exerted predominantly through actions of leptin within the CNS, most likely within the hypothalamus (Figure 3). The hypothalamic pathways involved in these actions are incompletely understood, although a role for melanocortin signaling pathways has been suggested. These central effects may be transmitted to the periphery through a variety of mechanisms, including the effects of altered appetite to decrease nutrient flux into the body and the effects of leptin on neuroendocrine or neural pathways. Leptin's effects on insulin sensitivity likely extend beyond those caused by alterations in food intake and nutrient flux, so actions via neuroendocrine or neural effectors are most likely involved as well. In many rodent models of obesity, including those due to leptin deficiency or resistance, increased glucocorticoids are an important mediator of both hyperphagia and insulin resistance, as seen through the beneficial effects of adrenalectomy (40). In $o b / o b$ mice, leptin replacement suppresses the activated hypothalamic-pituitary-adreno- 
cortical (HPA) axis, which may be an important component of leptin's action on insulin sensitivity, at least in rodents. Autonomic nerves may also be involved, as suggested by the effects of denervation to reduce leptin's action to promote glucose uptake into some muscle types (41). Major unanswered questions at this point include which signaling mechanism(s) and cellular targets in the periphery respond to the autonomic nerve output by which leptin affects metabolic pathways in relevant tissues, such as muscle, liver, and fat.

The second view of how leptin sensitizes to insulin involves direct effects at the level of insulin target tissues (Figure 3 ). In addition to the actions of leptin to modify metabolism via the brain, substantial data support the notion that leptin may have important effects through direct action on peripheral target cells, including $\beta$ cells, liver, muscle, and fat. Although initial surveys suggested that the ObRb isoform of the leptin receptor was not expressed in peripheral tissues, it now appears that receptor expression in such tissues occurs at biologically meaningful levels, as assessed by the ability to rapidly activate signaling events, including activation of STAT and MAPK pathways (42). Some evidence suggests that in tissues including muscle and $\beta$ cells, leptin promotes lipid oxidation and inhibits lipid synthesis, which would promote insulin sensitivity $(43,44)$. Current data do not allow determination of the relative importance of central versus peripheral actions of leptin in the metabolic actions of the hormone with certainty. Since leptin fails to reverse insulin resistance and lipid accumulation in mice with ventromedial hypothalamic lesions, and low-dose central administration of leptin has major metabolic effects without changing blood leptin levels, it seems likely that central actions are required, and that without them, the peripheral actions are at best limited. Since leptin expression is induced in tissues such as skeletal muscle after periods of feeding, it is possible that leptin produced locally has important metabolic actions as well (45). Tissue-specific knockout of leptin receptor isoforms may be helpful in clarifying this point. Even if, as we suspect, leptin's major actions are exerted within the CNS, we lack insight into the precise mechanism by which engaging central neural pathways rapidly changes the ability of leptin to regulate metabolic pathways in the periphery. This area will surely attract attention from the research community.

Unlike rodents, the few humans with leptin or leptin receptor mutations and obesity do not appear to have extraordinary degrees of insulin resistance, as assessed by hyperinsulinemia, and none have as yet been described with diabetes $(46,47)$. This difference may be related to the fact that in humans, unlike mice, leptin has little effect on the HPA axis. If leptin proves to have an important action on insulin sensitivity in humans, as it does in mice, then it will be important to determine the extent to which decreased leptin action, or leptin resistance, contributes to the insulin resistance of obesity in humans.

\section{Lipotoxicity and lipoatrophy: \\ two sides of the same coin?}

There are two additional ways in which alterations in the function of adipose tissue may influence glucose homeostasis. In one, increased adipose energy storage in obesity results in increased FFA flux to other tissues and increased triglyceride storage in these tissues, which promote insulin resistance and other adverse effects, referred to by some as "lipotoxicity." In the other, paradoxically, the absence of adipose tissue begets many of the same outcomes. How do we explain this paradox?

When the adipose depot is expanded, as in obesity, plasma FFAs become elevated, due, most likely, to increased release from the expanded adipose mass and probably also to impaired hepatic metabolism. Elevated FFAs impair the ability of insulin to suppress hepatic glucose output and to stimulate glucose uptake into skeletal muscle, as well as to inhibit insulin secretion from pancreatic $\beta$ cells. The defect in muscle may involve impaired activation of PI3K, possibly due to elevations in PKCO (48). An acquired loss of PI3K activation in muscle is also seen as a result of a high-fat diet (49). In humans, the triglyceride content of muscle correlates directly with insulin resistance, and the fatty acid composition of muscle phospholipids influences insulzzin sensitivity (50). We need to learn much more about the mechanisms that drive lipid accumulation in nonadipose tissues, and the mechanisms by which such lipotoxic impairment of function occurs. Recent studies in $\beta$ cells suggest that long-chain fatty acids may exert adverse effects via inducing overproduction of ceramide (51). It will be important to determine whether fatty acids alter gene expression through binding as ligands to transcription factors of the peroxisome proliferator-activated receptor (PPAR) family.

Severe deficiency or absence of fat occurs in patients with a heterogeneous group of disorders known as lipodystrophic diabetes, which, remarkably, is also associated with severe insulin resistance. It has been suggested that certain inherited disorders of insulin signaling pathways might account for both aspects of the disorder. However, thus far, no inherited defects in the insulin signaling pathway have been identified in these patients, and patients with insulin receptor mutations and insulin resistance do not have lipoatrophy. An alternative hypothesis, now supported by some published findings, holds that a primary deficiency of adipose tissue would cause severe insulin resistance. Two different mouse models in which fat is reduced or missing completely due to a disruption of adipogenesis have now been shown to have severe insulin resistance. In one case, leptin alone reversed the insulin resistance and diabetes (52). In the other, which shows more complete 
adipose loss, leptin replacement transiently ameliorated but did not reverse the disorder, whereas fat transplantation dramatically reversed insulin resistance and diabetes (53). Thus, it is clear that leptin and, possibly, other uncharacterized adipocyte products influence insulin sensitivity in the mouse.

That lipoatrophy causes insulin resistance by deficiency of secretory products of adipocytes, as opposed to the storage function alone, is also seen from studies of a "skinny" mouse, made deficient in adipose tissue through transgenic overexpression of leptin. Unlike lipoatrophic mice that lack leptin, these hyperleptinemic mice lacking visible adipose tissue have increased insulin sensitivity and do not accumulate triglyceride in muscles or liver (54). This may be due to the increased metabolic rate and body temperature in these mice, which result in increased substrate utilization instead of fat storage, as well as to effects of leptin to increase fatty acid oxidation (whether through the CNS or directly in peripheral tissues). Leptin increases fatty acid oxidation and decreases esterification in skeletal muscle (43) and in pancreatic islets (44). This appears to be due, at least in part, to regulation of the expression of genes involved in fatty acid metabolism and may be exerted directly at the level of the target tissues, as the effects are seen in muscle and islets exposed to leptin ex vivo. In islets of obese Zucker diabetic fatty rats with leptin receptor mutations, triglyceride content is 20 times greater than in lean controls and esterification capacity is increased three- to fourfold (55). Maneuvers that reduce islet fat content improve insulin secretion and prevent diabetes in these rats (55).

Insights from thiazoladinediones and PPAR $\gamma$. A major therapeutic advance in diabetes is the availability of thiazoladinediones (TZDs), a class of drugs that improve hyperglycemia, at least in part by improving insulin sensitivity (reviewed by Olefsky, this Perspective series, ref. 56). A breakthrough in understanding their mechanism of action came from the realization that the receptor for these drugs is the nuclear transcription factor PPAR $\gamma$, previously identified as a key transcription factor for adipogenesis. PPAR $\gamma$ is activated by TZDs, which bind to the ligand-binding domain and increase transactivation of target genes. The greatest expression of PPAR $\gamma$ is in adipocytes, although substantial expression is seen in some other tissues such as immune cells and vascular cells, and lower level expression of unknown significance is observed in tissues such as muscle and liver. The fact that TZDs act through $\operatorname{PPAR} \gamma$, and that PPAR $\gamma$ is predominantly expressed in fat, is a powerful new link between the adipocyte and insulin resistance. What exactly is the link?

The site of action and molecular targets of PPAR $\gamma$ that promote insulin sensitivity are poorly defined. TZDs may well act directly in muscle, which expresses PPAR $\gamma$ and contributes greatly to systemic insulin resistance. However, most interest heretofore has been in the direct effects of TZDs in fat, where PPAR $\gamma$ is very abundant, to reduce systemic insulin sensitivity. Thus, it has been suggested that TZDs act in fat, by unclear cellular mechanisms, to suppress release of FFAs or to decrease TNF signaling and TNF expression. TZDs reduce leptin expression and levels through actions on the $o b$ promoter, thereby promoting obesity. This mechanism, which has been difficult to demonstrate in humans taking TZDs, would not promote insulin sensitivity in any event. In rodents, TZDs increase the number of adipocytes, while decreasing their size. The increased number may be through well-described stimulation of adipogenesis, while the reduced size may be due to the lowering of ambient insulin levels, resulting in reduction in both lipid storage and antilipolysis. Smaller adipocytes are more insulin-sensitive, at least in terms of glucose transport, and this, as well as reduced release of TNF- $\alpha$ and other molecules from small adipocytes, may augment whole body insulin sensitivity. Finally, it is entirely possible that TZDs alter expression of an as yet unidentified adipocyte hormone that regulates insulin sensitivity.

The therapeutic effects of TZDs clearly suggest that $\operatorname{PPAR} \gamma$ regulates insulin sensitivity in diabetes. However, the mechanism is unknown and several additional key questions remain unanswered. First, do PPAR $\gamma$ dependent pathways fine-tune insulin sensitivity under normal circumstances? PPAR $\gamma$ is regulated both by expression of the protein and by the level of activating ligands; the endogenous ligands are unknown but are presumed to include FFAs or their derivatives. Once these molecules are identified, it will be important to learn what controls their levels in physiologic states, and whether their levels contribute to the etiology of insulin resistance. We are currently unable to answer these key questions, but with the answers will come important insights into the basis of insulin resistance. One experiment of nature strongly supports the idea that PPAR $\gamma$-dependent pathways regulate insulin sensitivity under normal circumstances in vivo in humans. A patient with severe insulin resistance has been found with an apparently dominant-negative mutation of PPAR $\gamma$. Surprisingly, this patient is not deficient in adipose tissue (in contrast to mice with PPAR $\gamma$ knockout), but the presence of severe insulin resistance strongly supports a critical role for PPAR $\gamma$ in the pathways responsible for insulin sensitivity in normal as well as diabetic individuals. Mice that are heterozygous for PPAR $\gamma$ gene deletion are protected from diet-induced insulin resistance, a finding that is difficult to explain.

\section{Conclusions and implications}

The relationship of obesity to insulin resistance and type 2 diabetes is a long-recognized phenomenon with fundamentally important scientific and clinical implications. The paradox that either absence or excess of adipose tissue causes insulin resistance highlights the 
complexity of this relationship and underscores the fact that adipose tissue undoubtedly subserves multiple functions. Advances over the last decade have expanded our understanding of the role of adipocytes in biology, and this has begun to provide mechanistic insights into the causal relationship between obesity and diabetes. It is now clear that adipocytes function as endocrine glands with wide-reaching effects on other organs including the brain. The release of a wide variety of molecules including hormones such as leptin, cytokines such as TNF- $\alpha$, and substrates such as FFAs allows the adipose organ to play a major regulatory role in energy balance and glucose homeostasis. Current challenges on the horizon include (a) discovering additional adipocyte-secreted products which are central to these processes; (b) deepening our understanding of the cellular and molecular mechanisms underlying insulin sensitivity and secretion, including previously unidentified pathways such as those mediated by nuclear receptors such as PPAR $\gamma$; and (c) exploring the possibility of divergence among different insulin-mediated processes, such that those driving adipogenesis and fat storage may actually be augmented, at least in some tissues, in insulin-resistant states.

Most likely, the complex orchestration of the relationship between adipose tissue, insulin action, and glucose homeostasis evolved out of survival needs to maintain fuel supplies when food was scarce. Now in times of plenty in Western society, obesity with its accompanying morbidities has reached epidemic proportions and the need for scientific advances to identify new therapeutic approaches could not be more acute. The challenge to use the increasing repertoire of adipocyte functions to shift the equation of energy intake and utilization toward reduced fat storage holds great opportunities to alter the course of human disease.

\section{Acknowledgments}

This work was supported by grants from the NIH (RO1 DK-43051, RO1 DK-28082, and PO1 DK-56116) and a grant from Eli Lilly and Co. The authors recognize many additional contributions to the field which they were unable to cite due to a limitation in the number of references. The authors thank Young-Bum Kim for expert assistance.

1. Reaven, G.M. 1995. Pathophysiology of insulin resistance in human disease. Physiol. Rev. 75:473-486.

2. Paradis, S., and Ruvkun, G. 1998. Caenorhabditis elegans Akt-PKB transduces insulin receptor-like signals from AGE-1 PI3 kinase to the DAF-16 transcription factor. Genes Dev. 12:2488-2498.

3. White, M.F. 1998. The IRS-signaling system: a network of docking proteins that mediate insulin and cytokine action. Recent Prog. Horm. Res. 53:119-138.

4. Staubs, P.A., Nelson, J.G., Reichart, D.R., and Olefsky, J.M. 1998. Plateletderived growth factor inhibits insulin stimulation of insulin receptor substrate-1-associated phosphatidylinositol 3-kinase in 3T3-L1 adipocytes without affecting glucose transport. J. Biol. Chem. 273:25139-25147.

5. Czech, M.P., and Corvera, S. 1999. Signaling mechanisms that regulate glucose transport. J. Biol. Chem. 274:1865-1868.

6. Wang, Q., et al. 1999. Protein kinase B/Akt participates in GLUT4 translocation by insulin in L6 myoblasts. Mol. Cell. Biol. 19:4008-4018.

7. Kitamura, T., et al. 1998. Requirement for activation of the serine-threonine kinase akt (Protein kinase B) in insulin stimulation of protein synthesis but not of glucose transport. Mol. Cell. Biol. 18:3708-3717.

8. Kotani, K., et al. 1998. Requirement of atypical protein kinase clambda for insulin stimulation of glucose uptake but not for Akt activation in 3T3-L1 adipocytes. Mol. Cell. Biol. 18:6971-6982.

9. Kitamura, T., et al. 1999. Insulin-induced phosphorylation and activation of cyclic nucleotide phosphodiesterase $3 \mathrm{~B}$ by the serine-threonine kinase Akt. Mol. Cell. Biol. 19:6286-6296.

10. Kim, J.B., et al. 1998. Nutritional and insulin regulation of fatty acid synthetase and leptin gene expression through ADD-1/SREBP1. J. Clin. Invest. 101:1-9.

11. Shimomura, I., et al. 1999. Insulin selectively increases SREBP-1c mRNA in the livers of rats with streptozotocin-induced diabetes. Proc. Natl. Acad. Sci. USA. 96:13656-13661.

12. Foretz, M., Guichard, C., Ferré, P., and Foufelle, F. 1999. Sterol regulatory binding element binding protein-1c is a major mediator of insulin action on the hepatic expression of glucokinase and lipogenesis-related genes. Proc. Natl. Acad. Sci. USA. 96:12737-12742.

13. Kops, G.J., and Burgering, B.M. 1999. Forkhead transcription factors: new insights into protein kinase B (c- akt) signaling. J. Mol. Med. 77:656-665.

14. Rondinone, C.M., et al. 1997. Insulin receptor substrate (IRS) 1 is reduced and IRS-2 is the main docking protein for phosphatidylinositol 3-kinase in adipocytes from subjects with non-insulin-dependent diabetes mellitus. Proc. Natl. Acad. Sci. USA. 94:4171-4175.

15. Kim, Y.B., Nikoulina, S.E., Ciaraldi, T.P., Henry, R.R., and Kahn, B.B. 1999. Normal insulin-dependent activation of Akt/protein kinase B, with diminished activation of phosphoinositide 3-kinase, in muscle in type 2 diabetes. J. Clin. Invest. 104:733-741.

16. Goldstein, B.J., Ahmad, F., Ding, W., Li, P.M., and Zhang, W.R. 1998. Regulation of the insulin signalling pathway by cellular protein-tyrosine phosphatases. Mol. Cell. Biochem. 182:91-99.

17. Goldstein, B.J., Bittner-Kowalczyk, A., White, M.F., and Harbeck, M. 2000. Tyrosine dephosphorylation and deactivation of insulin receptor substrate- 1 by protein-tyrosine phosphatase $1 \mathrm{~B}$. Possible facilitation by the formation of a ternary complex with the grb2 adaptor protein. J. Biol. Chem. 275:4283-4289.

18. Elchebly, M., et al. 1999. Increased insulin sensitivity and obesity resistance in mice lacking the protein tyrosine phosphatase-1B gene. Science. 283:1544-1548.

19. Klaman, L.D., et al. 2000. Increased energy expenditure, decreased adiposity, and tissue-specific insulin sensitivity in protein-tyrosine phosphatase 1B (PTP1B)-deficient mice. Mol. Cell. Biol. 20:5479-5489.

20. Goodyear, L.J.,et al. 1995. Insulin receptor phosphorylation, insulin receptor substrate-1 phosphorylation, and phosphatidylinositol 3kinase activity are decreased in intact skeletal muscle strips from obese subjects. J. Clin. Invest. 95:2195-2204.

21. Shepherd, P.R., and Kahn, B.B. 1999. Glucose transporters and insulin action: implications for insulin resistance and diabetes mellitus. $N$. Engl. J. Med. 341:248-257.

22. Kraegen, E.W., James, D.E., Jenkins, A.B., and Chisholm, D.J. 1985. Doseresponse curves for in vivo insulin sensitivity in individual tissues in rats. Am. J. Physiol. 248:E353-E362.

23. Shepherd, P.R., et al. 1993. Adipose cell hyperplasia and enhanced glucose disposal in transgenic mice overexpressing GLUT4 selectively in adipose tissue. J. Biol. Chem. 268:22243-22246.

24. Tozzo, E., Gnudi, L., and Kahn, B.B. 1997. Amelioration of insulin resistance in streptozotocin diabetic mice by transgenic overexpression of GLUT4 driven by an adipose-specific promoter. Endocrinology. 138:1604-1611.

25. de Souza, C.J., Hirshman, M.F., and Horton, E.S. 1997. CL-316,243, a beta3-specific adrenoceptor agonist, enhances insulin-stimulated glucose disposal in nonobese rats. Diabetes. 46:1257-1263.

26. Weyer, C., Tataranni, P.A., Snitker, S., Danforth, E., Jr., and Ravussin, E. 1998. Increase in insulin action and fat oxidation after treatment with CL 316,243, a highly selective beta3-adrenoceptor agonist in humans. Diabetes. 47:1555-1561.

27. Colditz, G.A., et al. 1990. Weight as a risk factor for clinical diabetes in women. Am. J. Epidemiol. 132:501-513.

28. Kissebah, A.H., and Krakower, G.R. 1994. Regional adiposity and morbidity. Physiol. Rev. 74:761-811. 
29. Patti, M.E., and Kahn, C.R. 1996. Lessons from transgenic and knockout animals about noninsulin-dependent diabetes mellitus. Trends Endocrinol. Metab. 7:311-319.

30. Bujalska, I.J., Kumar, S., and Stewart, P.M. 1997. Does central obesity reflect "Cushing's disease of the omentum"? Lancet. 349:1210-1213.

31. Deslypere, J.P., Verdonck, L., and Vermeulen, A. 1985. Fat tissue: a steroid reservoir and site of steroid metabolism. J. Clin. Endocrinol. Metab. 61:564-570.

32. Peraldi, P., and Spiegelman, B. 1998. TNF-alpha and insulin resistance: summary and future prospects. Mol. Cell. Biochem. 182:169-175.

33. Hotamisligil, G.S. 1999. The role of TNFalpha and TNF receptors in obesity and insulin resistance. J. Intern. Med. 245:621-625.

34. Friedman, J.M. 2000. Obesity in the new millennium. Nature. 404:632-634

35. Flier, J.S. 1998. What's in a name? In search of leptin's physiologic role. J. Clin. Endocrinol. Metab. 83:1407-1413.

36. Elmquist, J.K., Maratos-Flier, E., Saper, C.B., and Flier, J.S. 1998. Unraveling the central nervous system pathways underlying responses to leptin. Nat. Neurosci. 1:445-450.

37. Halaas, J.L., et al. 1995. Weight-reducing effects of the plasma protein encoded by the obese gene. Science. 269:543-546.

38. Campfield, L.A., Smith, F.J., Guisez, Y., Devos, R., and Burn, P. 1995. Recombinant mouse OB protein: evidence for a peripheral signal linking adiposity and central neural networks. Science. 269:546-549.

39. Pelleymounter, M.A., et al. 1995. Effects of the obese gene product on body weight regulation in ob/ob mice. Science. 269:540-543.

40. Bray, G.A. 1985. Autonomic and endocrine factors in the regulation of food intake. Brain Res. Bull. 14:505-510.

41. Kamohara, S., Burcelin, R., Halaas, J.L., Friedman, J.M., and Charron, M.J. 1997. Acute stimulation of glucose metabolism in mice by leptin treatment. Nature. 389:374-377.

42. Kim, Y.-B., Uotani, S., Pierroz, D.D., Flier, J.S., and Kahn, B.B. 2000. In vivo administration of leptin activates signal transduction directly in insulin sensitive tissues: overlapping but distinct pathways from insulin. Endocrinology. 141:2328-2339.

43. Muoio, D.M., Dohm, G.L., Fiedorek, F., Jr., Tapscott, E.B., and Coleman,
R.A. 1997. Leptin directly alters lipid partitioning in skeletal muscle. Diabetes. 46:1360-1363.

44. Shimabukuro, M., et al. 1997. Direct antidiabetic effect of leptin through triglyceride depletion of tissues. Proc. Natl. Acad. Sci. USA. 94:4637-4641.

45. Wang, J., Liu, R., Hawkins, M., Barzilai, N., and Rossetti, L. 1998. A nutrient-sensing pathway regulates leptin gene expression in muscle and fat. Nature. 393:684-688.

46. Montague, C.T., et al. 1997. Congenital leptin deficiency is associated with severe early-onset obesity in humans. Nature. 387:903-908.

47. Clement, K., et al. 1998. A mutation in the human leptin receptor gene causes obesity and pituitary dysfunction. Nature. 392:398-401.

48. Griffin, et al. 1999. Free fatty acid-induced insulin resistance is associated with activation of protein kinase $\mathrm{C}$ theta and alterations in the insulin signaling cascade. Diabetes. 48:1270-1274.

49. Zierath, J.R., Houseknecht, K.L., Gnudi, L., and Kahn, B.B. 1997. Highfat feeding impairs insulin-stimulated GLUT4 recruitment via an early insulin-signaling defect. Diabetes. 46:215-223.

50. Borkman, M., et al. 1993. The relation between insulin sensitivity and the fatty-acid composition of skeletal-muscle phospholipids. N. Engl. J. Med. 328:238-244.

51. Shimabukuro, M., et al. 1998. Lipoapoptosis in beta-cells of obese prediabetic $\mathrm{fa} / \mathrm{fa}$ rats. Role of serine palmitoyltransferase overexpression. $J$. Biol. Chem. 273:32487-32490.

52. Shimomura, I., Hammer, R.E., Ikemoto, S., Brown, M.S., and Goldstein, J.L. 1999. Leptin reverses insulin resistance and diabetes mellitus in mice with congenital lipodystrophy. Nature. 401:73-76.

53. Gavrilova, O., et al. 2000. Surgical implantation of adipose tissue reverses diabetes in lipoatrophic mice. J. Clin. Invest. 105:271-278.

54. Ogawa, Y., et al. 1999. Increased glucose metabolism and insulin sensitivity in transgenic skinny mice overexpressing leptin. Diabetes. 48:1822-1829.

55. Unger, R.H., Zhou, Y.T., and Orci, L. 1999. Regulation of fatty acid homeostasis in cells: novel role of leptin. Proc. Natl. Acad. Sci. USA. 96:2327-2332.

56. Olefsky, J.M. 2000. Treatment of insulin resistance with peroxisome proliferator-activated receptor $\gamma$ agonists. J. Clin. Invest. 106:467-472. 